\title{
Single-incision Robotic Cholecystectomy: Initial Experience and Results
}

\author{
Yuan Yu Cheng, M.D., Hyung Ook Kim, M.D., Ph.D., Byung Ho Son, M.D., Ph.D., Jun Ho Shin, M.D., Ph.D., \\ Sung Ryol Lee, M.D., Ph.D. \\ Department of Surgery, Kangbuk Samsung Hospital, Sungkyunkwan University School of Medicine, Seoul, Korea
}

\begin{abstract}
Purpose: Continued efforts to reduce the invasiveness of conventional cholecystectomy techniques have resulted in the development of single-incision cholecystectomy. However, a single-port approach has significant limitations associated with proper triangulation and instrument crowding and collisions. Although the da Vinci Single-Site robotic system has been proposed to overcome these problems, objective evidence of the feasibility and ergonomics of single-incision robotic cholecystectomy (SIRC) is insufficient. Therefore, the present study aimed to evaluate the feasibility and efficacy of SIRC by using objective data obtained from consecutive patients who underwent surgery with the single-incision robotic platform performed by a single surgeon.
\end{abstract}

Methods: Forty patients who underwent SIRC between August 2014 and December 2015 were identified. Demographic, perioperative, and postoperative data were collected retrospectively.

Results: The mean docking time was 10.82 $\pm 4.85 \mathrm{~min}$ (range, 4 30 min). The mean console time was $49.63 \pm 10.82 \mathrm{~min}$ (range, 24 90 min). None of the patients required an additional laparoscopic arm, an additional robotic arm, or conversion to conventional laparoscopic cholecystectomy.

Conclusion: SIRC can provide a safe operative procedure, good operative results, and high patient satisfaction, and cause less surgeon fatigue. Therefore, our study results indicate that SIRC is feasible and favorable for both patients and physicians.

Keywords: Laparoscopy, Cholecystectomy, Minimally Invasive surgical procedures
Received July 12, 2016

Revised 1st October 19, 2016

2nd October 20, 2016

3rd October 21, 2016

Accepted October 23, 2016

Corresponding author

Sung Ryol Lee

Department of Surgery, Kangbuk Samsung Hospital, Sungkyunkwan University School of Medicine, 29, Saemunan-ro, Jongno-gu, Seoul 03181, Korea

Tel: +82-2-2001-2001

Fax: +82-2-2001-2131

E-mail: Sungryol.lee@samsung.com

\section{INTRODUCTION}

Since the 1980s, laparoscopic cholecystectomy has been widely used and has become a basic cholecystectomy procedure. Continued efforts to further reduce the invasiveness of this procedure have resulted in the development of singleincision laparoscopic cholecystectomy (SILC). ${ }^{1-3}$

Navarra performed the first single-incision laparoscopic cholecystectomy procedure through the trans-umbilical approach in 1997. In recent years, literature related to this tech- nology has accelerated to accumulate. ${ }^{4}$ While positive studies on the technology have been reported, ${ }^{5-7}$ the single-port approach still presents significant limitations related to proper triangulation, instrument crowding, and collisions. ${ }^{8-10}$ Such limitations complicate surgical procedures and tend to entice surgeons to proceed with a simple multi-incision approach.

The recently developed Da Vinci Single-Site robotic system (Intuitive Surgical, Sunnyvale, CA) has been introduced to overcome these problems. This set of instruments is designed to be used with the Da Vinci Si Surgical System (Intuitive 
Surgical, Sunnyvale, CA) in single-incision laparoscopic surgery. ${ }^{11,12}$ The semi-rigid instruments are delivered into the abdominal cavity by curved cannulae that are inserted into a specific port through a single fascial incision. This system electrically reverses robotic arms, allowing surgeon's twohanded movements to be as natural as usual. The electrical crossover helps minimizing collision between instruments through a 2 to $3 \mathrm{~cm}$ incision and the robotic system stably adjusts the camera and other instruments during cholecystectomy. Although the Single-Site technology is non-wristed and, unlike other conventional robotic instruments, only provides rotation, the ergonomics are nevertheless greatly improved. ${ }^{13}$ Therefore, the present study is designed to evaluate the feasibility and efficacy of single-incision robotic cholecystectomy using the single-incision robotic platform in 40 consecutive cases, based on objective data from a single surgeon.

\section{MATERIALS AND METHODS}

Forty patients who underwent single-incision robotic cholecystectomy (SIRC) between August 2014 and December 2015 were enrolled. The patients included in this study were selected from the entire cohort of patients who underwent SIRC. The demographic, perioperative, and postoperative data from the follow-up period were retrospectively collected for all $\mathrm{pa}^{-}$ tients who had undergone SIRC. The patient selection for the surgical method was based solely on the patient's preference for SIRC. The initial inclusion criteria were patients between the age of 18 and 80 years with symptomatic cholelithiasis, polyps, or chronic cholecystitis. The exclusion criteria were: (1) severe acute cholecystitis, cholangitis, or common bile duct stones; (2) biliary pancreatitis; (3) cirrhosis; (4) bleeding disorders; (5) suspicious or proven malignancy; (6) history of abdominal surgery; (7) mental illness; (8) pregnancy; and (9) rejection of SIRC.

Preoperative evaluations involved biliary ultrasounds, computed tomographies, and routine blood tests. Preoperative cholangiographies were not routinely performed; however, in cases of suspected bile duct stones, magnetic resonance cholangio-pancreatographies (MRCPs) were performed preoperatively.

Anesthesia times were recorded from induction to recovery, and operation times were recorded from incision to wound closure. Patient preparations followed the same steps as for conventional laparoscopic cholecystectomy. Abdomens were shaved, prepped, and draped in the usual sterile fashion, and patients were held in a supine position with both arms attached to the body. The initial access involved a $2.5 \mathrm{~cm}$ midline umbilical incision down to the level of fascia. In all cases, fasciae were incised longitudinally. Prior to port insertion, the port was everted in the same way as when using the regular Da Vinci Si Surgical System. This method allowed the assist cannula to be located on the right side of the insufflation adaptor, giving the assistant a wider range of motion. Using an atraumatic clamp, the port was grasped just above the lower rim and was softly inserted with lubrication. The operation was performed in a partial reverse Trendelenburg position. The laparoscopic grasper was inserted, and the fundus of the Gall bladder was pulled for confirmation of the anatomy and movement of the gall bladder. Before docking, all surgical instruments were removed and the robotic arm was placed in position. The operation cart was moved to the patient. The camera was inserted vertically. A curved cannula with lubricant was placed into the designated lumen, where the assistant held the external rim to prevent the port from slipping. The scope should always show the cannula tip to avoid unintentional organ damage. The cannula was guided near to the target organ and was then held still to allow for docking. This was achieved by holding the cannula still in one hand, while the other hand brought and mounted the arm to the cannula. Before selecting the cannula, the body surface area (BSA) was calculated to avoid a tissue injury from the long arm and a loss of power from the short arm. If the BSA was greater than 1.8 $\mathrm{m}^{2}$, a $300-\mathrm{mm}$ curved cannula was selected, while a $250-\mathrm{mm}$ curved cannula was selected if the BSA was smaller than 1.8 $\mathrm{m}^{2}$. Finally, robotic surgical instruments were inserted through the designated cannula, and the position for surgery was completed. The assistant's cannula, which was used to hold and lift the fundus of the gall bladder using a long grasping forceps, was inserted last. The endoscope was guided by the surgeon under the grasper. This resulted in both retraction of the gall bladder and exposure of Calot's triangle.

The postoperative pain scores based on the Numerical Pain Rating Scale were collected retrospectively, using a computerized database system operated by an independent nurse at the time of the study. ${ }^{14,15}$ Pain management was performed with non-steroidal anti-inflammatory agents (ibuprofen $800 \mathrm{mg}$ during $24 \mathrm{~h}$ ) administered at the patients' request. All of the surgeries were performed by a single surgeon with a rich $\mathrm{ex}^{-}$ perience of laparoscopic cholecystectomy.

All the statistical analyses were performed with STATA version 14.0 (Stata Corp LP, College Station, TX). The study was approved by the Ethical Review Board of our hospital.

\section{RESULTS}

The patients' ages ranged between 28 and 60 years (mean age: $41.80 \pm 9.35$ years) (Table 1). Their body mass index (BMI) ranged widely from 19.1 to $32.1 \mathrm{~kg} / \mathrm{m}^{2}$ (mean: $24.0 \pm 3.28 \mathrm{~kg}$ ) $\mathrm{m}^{2}$ ), and their BSA ranged from 1.33 to $2.33 \mathrm{~m}^{2}$ (mean: $1.74 \pm$ 
Table 1. Patient demographics

\begin{tabular}{|c|c|}
\hline & $\mathrm{N}=\mathbf{4 0}$ \\
\hline Sex (M:F) & $23: 17$ \\
\hline Age (year) & $39.5(28 \sim 60)$ \\
\hline BMI $\left(\mathrm{kg} / \mathrm{m}^{2}\right)$ & $24.03(19.07 \sim 31.10)$ \\
\hline BSA $\left(\mathrm{m}^{2}\right)$ & $1.74(1.33 \sim 2.33)$ \\
\hline ASA score & \\
\hline 1 & 28 \\
\hline 2 & 12 \\
\hline 3 & 0 \\
\hline Asymptomatic : Symptomatic & $22: 18$ \\
\hline Preoperative USG & 11 \\
\hline Chronic cholecystitis & 18 \\
\hline GB polyp & 2 \\
\hline GB polyp \& stone & 9 \\
\hline Cholelithiasis & \\
\hline Preoperative CT & 18 \\
\hline Chronic cholecystitis & 18 \\
\hline GB polyp & 4 \\
\hline Cholelithiasis & 20 \\
\hline Pathologic diagnosis & 11 \\
\hline Chronic cholecystitis & 9 \\
\hline Cholesterol polyp & \\
\hline Adenomatous polyp & \\
\hline - Body Mass & \\
\hline
\end{tabular}

$B M I=$ Body Mass Index; $B S A=$ Body Surface Area; $A S A=$ American Society of Anesthesiologists; USG $=$ Ultrasonography; $C T=$ Computed tomography; $\mathrm{GB}=$ Gall bladder.

0.21 . None of the patients had received previous upper abdominal surgery; however, five (12.5\%) had undergone prior lower abdominal surgery. The primary indication for surgery was gall bladder polyps $(18 / 40,45.0 \%)$, followed by chronic cholecystitis $(11 / 40,27.5 \%)$, symptomatic cholelithiasis $(9 / 40,22.5 \%)$, and gall bladder polyps and stones (2/40, 5.0\%). Eight cases (20.0\%) presented significant adhesions in Calot's triangle that required prior adhesiolysis. Some patients $(15 / 40,37.5 \%)$ had concomitant disease or were receiving medication for diabetes mellitus, hypertension, or hypothyroidism. The physical status of the patients was classified in accordance with the American Society of Anesthesiologists score: 28 patients were ASA 1, 12 were ASA 2, and 0 were ASA 3 (mean, $1.30 \pm 0.46$ ).

All SIRCs were performed by the same operator, and were conducted with the same team in the same room. The results
Table 2. Operation results

\begin{tabular}{|c|c|}
\hline & $\mathrm{N}=40$ \\
\hline \multicolumn{2}{|l|}{ Operation time $(m i n u t e)^{*}$} \\
\hline Docking time & $10.82 \pm 4.85$ \\
\hline Console time & $49.63 \pm 13.17$ \\
\hline Total operation & $97.25 \pm 20.03$ \\
\hline Bleeding (ml) & $5.77 \pm 1.88$ \\
\hline Completed as single site & 40 \\
\hline Conversion & 0 \\
\hline \multicolumn{2}{|l|}{ Pain score } \\
\hline After 6 hour & $4.77 \pm 1.25$ \\
\hline After 1 day & $1.95 \pm 1.10$ \\
\hline Follow-up days & $0.25 \pm 0.43$ \\
\hline Hospital POD & $2.05 \pm 0.87$ \\
\hline
\end{tabular}

POD $=$ Postoperative day, ${ }^{*}$ The results for thr entire patients.

are summarized in Table 2. The timings of the operation were determined as follows: (1) Docking time: The time from the moment the port is inserted until the second robotic arm is installed in the cannula; (2) Console time: The actual procedure time from the moment the surgeon operates the surgical instrument to when it is collected; and (3) Operation time: The time from skin incision to completion of skin closure. The mean docking time was $10.82 \pm 4.85$ min (range: 4 30 min, Fig. 1), the mean console time was $49.63 \pm 10.82$ min (range: 24 90 min, Fig. 2), and the mean operation time was 97.25 20.03 min (range: 60 150 min, Fig. 3). There was no meaningful difference in the operation time of the patients with GB polyps and those with GB stones $(96.94 \pm 21.08$ min vs $96.75 \pm$ $18.80 \mathrm{~min}$, Table 2).

During the SIRC, none of the operations required an additional laparoscopic arm, an additional robotic arm, or conversion to conventional laparoscopic cholecystectomy on account of bleeding and adhesion. There were no open conversions. The mean number of postoperative days (POD) for all patients was $2.05 \pm 0.87 \mathrm{~d}$ (range: $1.0 \sim 4.0 \mathrm{~d}$ ). The hospitalization was extended in cases of postoperative fever or for personal reasons (typically related to the individual's insurance).

There were not any interferences between the robotic instruments or robot-related problems during operations. Moreover, there were no intraoperative complications, such as major intraoperative bleedings or bile duct injuries. In addition, there was no bile leak from the gall bladder during its detachment from the liver. The mean estimated blood loss was $5.77 \pm 1.88$ $\mathrm{ml}$, and all incisions were $2.5 \mathrm{~cm}$ in length. The estimation of the postoperative pain was based on the Numerical Pain 


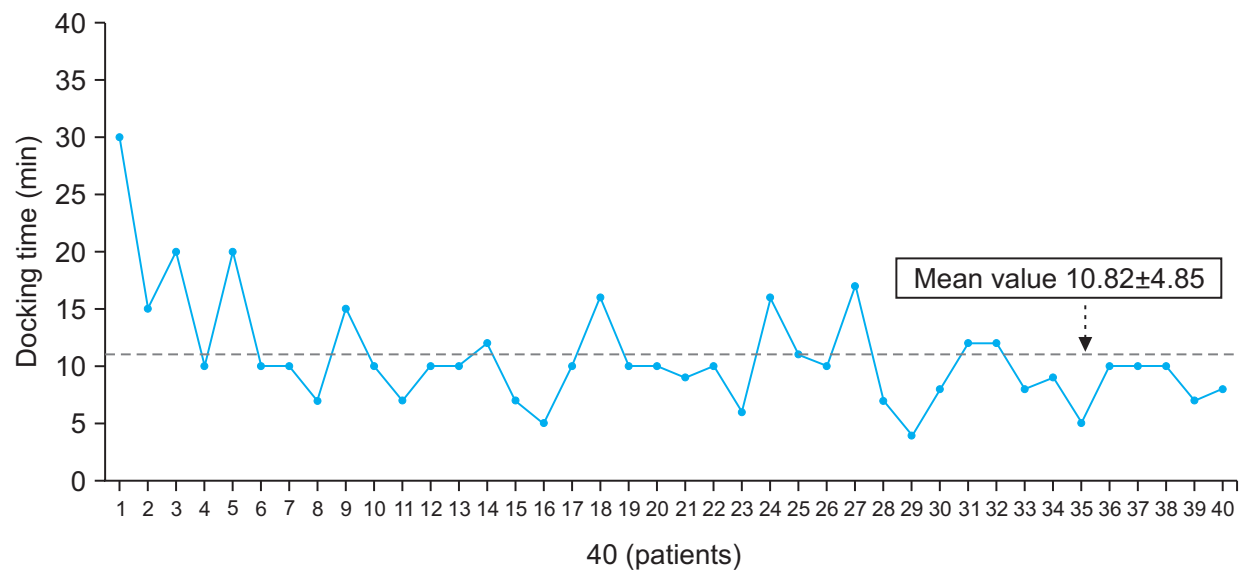

Fig. 1. Pattern of docking time.

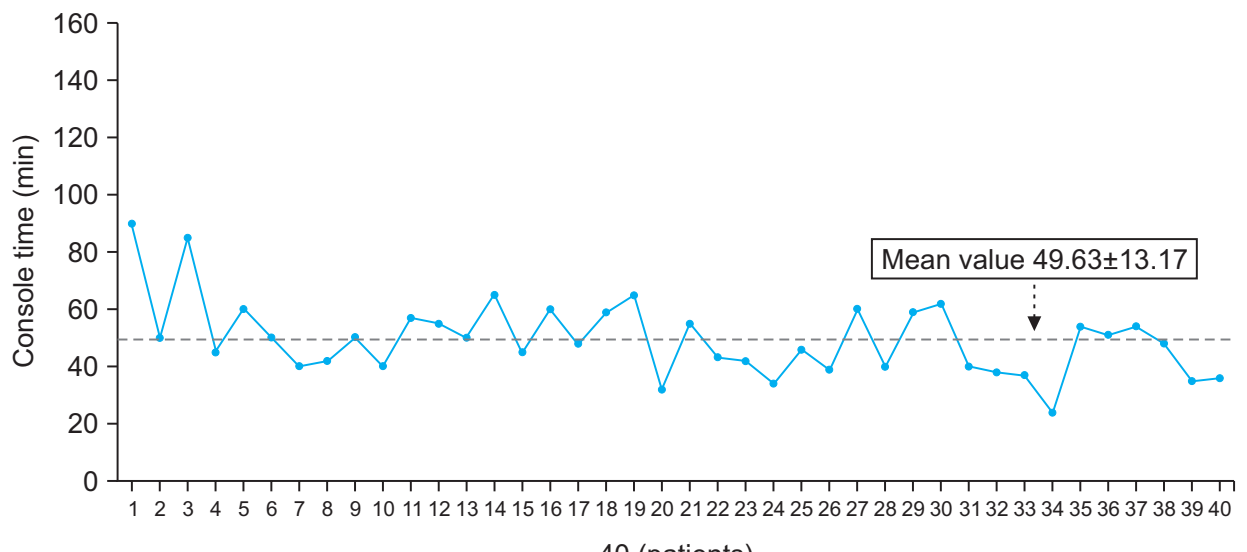

40 (patients)

Fig. 2. Pattern of console time.

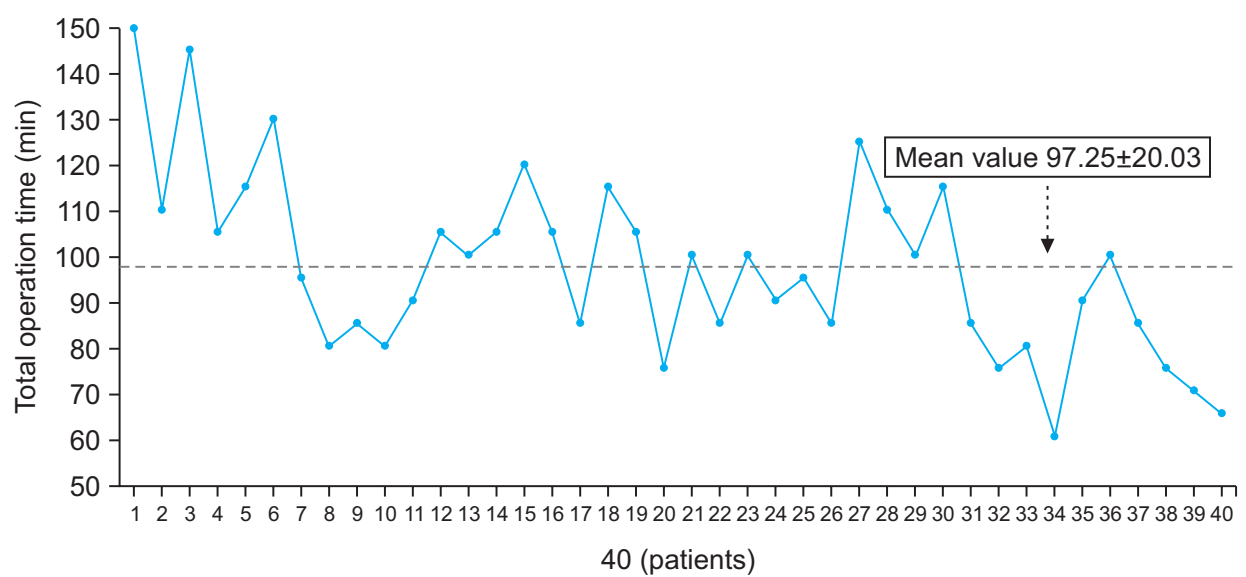

Fig. 3. Pattern of total operation time.

Rating Scale. ${ }^{14,15}$ The mean pain score was $4.77 \pm 1.25$ (range: 3 7) $6 \mathrm{hr}$ after the operation, $1.95 \pm 1.10$ (range: 0.0 5.0) on postoperative day 1 , and $0.25 \pm 0.43$ (range: $0 \sim 1$ ) 2 weeks after the surgery (Table 2). The patients were followed-up for up to 3 months after the operation. All patients were fully recovered from postoperative symptoms such as surgical pains and wounds, and were satisfied with outcome of operations.

\section{DISCUSSION}

The number of SILC cases has been increasing and related data have been accumulating. However, it is too early to approve it as a new gold standard for the treatment of Gall stone disease. Although several studies have reported the safety and suitability of SILC, ${ }^{5-7}$ the possibility that complications associ- 
ated with surgery may increase is still concern, especially the possibility of biliary duct injuries. ${ }^{9,13,16}$ Therefore, continuous efforts to overcome technical constraints have been made. The primary challenge for single-port laparoscopic surgery is to secure a safe area for effective dissection and traction while minimizing damage to surrounding tissue. Various methods have been proposed for effective traction and suture of the gall bladder fundus by using the Keith needle or hook has been attempted. ${ }^{17,18}$ In addition, despite the introduction of flexible instruments and camera systems to address this problem, collision of the laparoscopic instruments remains a problem in manipulating tissues or in delicate movements. For this reason, this disadvantage tends to increase the operating time and learning curve.

The Da Vinci Single-Site robotic system has been introduced to overcome these problems. This set of instruments is designed to be used with the Da Vinci Si Surgical System in single-incision surgery. ${ }^{11,12}$ In SIRC, the flexible robotic arm crosses the other arm through the port, and the robot system electrically reverses the robotic arm to the right and to the left so that the surgeon's hand motion is natural. This electrical crossover helps the cholecystectomy to minimize the collision between the instruments through a 2 to $3 \mathrm{~cm}$ incision and the robotic system stably adjusts the camera and instruments during surgery.

However, despite the excellent outcomes of the current SIRC method, objective evidence is lacking. Therefore, this study aimed to consider several aspects of SIRC objectively.

The first important aspect was the mode of retraction for fine dissection. In SILC, several methods of gallbladder retracting-including sutures, clips, and magnets-have been described. ${ }^{19,20}$ In this study, however, none of the previous methods have been proven to be necessary. The retractor grasper was inserted via the accessary channel of the reverse port, moved under the rigid scope, advanced along the same axis, and entered into the surgical field. The assistant did not have to keep the grasper in tension; however, it merely supported it gently to keep the gallbladder steady as the camera arm was moving. Furthermore, the grasper can only move along its longitudinal axis, which allows the fundus of Gall bladder to move toward the patient's head or to the coccyx to easily obtain the optimal surgical field. By establishing a "reverse port" before insertion, the assistant can obtain a wider range of motion, and the surgeon a finer Calot's triangle.

The second important challenge is to achieve an excellent view. The camera of the Da Vinci Si Surgical System offers a better view during SIRC than any other endoscope. Although intraoperative cholangiography is feasible during SIRC, it was not performed in this study, as the "reverse port" technique provided a good view and a definite Calot's triangle. Addition- ally, as a result of the cannula selection using the BSA standards (300 mm for a BSA $>1.8 \mathrm{~m}^{2}, 250 \mathrm{~mm}$ for a BSA $<1.8 \mathrm{~m}^{2}$ ) and the good visibility conditions, this study did not report any tissue injury. Thanks to the advantages described above, the cases in this study did not require any additional laparoscopic arm, additional robotic arm, or conversion to conventional laparoscopic cholecystectomy. Additionally, there were no cases of open conversion, and no intraoperative or postoperative complications such as tissue injury or major bleeding (mean blood loss: $5.77 \pm 1.88 \mathrm{ml}$ ).

The third issue was the operation time. In a study by Rivas et al., ${ }^{8}$ the average surgery time for the first 50 cases of SILC was 73 min (range: 35 120). In the study by Morel et al.," the average total operation time for SIRC was $91.1 \mathrm{~min}$, the console time was $50.9 \mathrm{~min}$, and the docking time was $6.6 \mathrm{~min}$. The present study showed a total operation time of $97.25 \mathrm{~min}$, a console time of $49.63 \mathrm{~min}$, and a docking time of $10.82 \mathrm{~min}$. The main difference between the SILC and SIRC techniques lies in the docking time. Unlike SILC, SIRC requires an average time of 10 min for docking. However, after the initial period (1 20 cases, Fig. 1), the docking time showed a consistent pattern of 5 8 $\mathrm{min}$, and the console time was reduced to 40 min with the accumulation of experience. This pattern suggests that SIRCs' long total operation time can be overcome.

The fourth issue are the postoperative hospital d. Konstantinos et al. ${ }^{12}$ reported that the mean PODs for SIRC were 23.84 \pm 10.27 h. Morel et al. ${ }^{21}$ reported mean PODs of $2.4 \mathrm{~d}$. In this study, the PODs for all patients were $2.05 \pm 0.87 \mathrm{~d}$ (range: $1 \sim 4$ d). However, in our study, the higher number of PODs after SIRC were not due to complications but to the insurance system, which offered inexpensive admission charges.

The fifth aspect were the patient-related factors of the pain and cosmetic results. All patients in our study had a $2.5 \mathrm{~cm}^{-}$ long axis incision at the natural umbilicus. Three months after the operation, none of them-except for two very thin patients with a low BMI-could find any scar, and none of the patients presented any complications such as incisional hernia. All of the patients were satisfied with their umbilical scar. The pain score on POD 1 was low $(1.95 \pm 1.10)$, and the pain nearly completely disappeared in the immediate follow-up days $(0.25 \pm$ 0.43) after discharge. However, it should be noted that painkillers were administered to all patients in this study.

In conclusion, SIRC yielded several benefits, including the lack of need for an additional port, no complications and conversions, a good completion rate, and a low pain score. Therefore, like other single-incision cholecystectomy techniques, SIRC is suitable for symptomatic cholelithiasis, gallbladder polyps, and cholecystitis. However, questions remain about the increased cost and relatively numerous exclusion criteria. Future studies should examine the true benefits of SIRC by 
conducting larger randomized controlled trials involving conventional laparoscopic and multiport robotic approaches.

\section{REFERENCES}

1) Vidal $O$, Valentini $M$, Ginesta $C$, et al. Single-incision versus standard laparoscopic cholecystectomy: comparison of surgical outcomes from a single institution. J Laparoendosc Adv Surg Tech A 2011;21:683-686.

2) Hagen ME, Wagner OJ, Thompson K, et al. Supra-pubic single incision cholecystectomy. J Gastrointest Surg 2010;14:404-407.

3) Lee PC, Lo C, Lai PS, et al. Randomized clinical trial of singleincision laparoscopic cholecystectomy versus minilaparoscopic cholecystectomy. Br J Surg 2010;97:1007-1012.

4) Navarra G, Pozza E, Occhionorelli S, Carcoforo P, Donini I. Onewound laparoscopic cholecystectomy. Br J Surg 1997;84:695.

5) Hodgett SE, Hernandez JM, Morton CA, Ross SB, Albrink M, Rosemurgy AS. Laparoendoscopic single site (LESS) cholecystectomy. J Gastrointest Surg 2009;13:188-192.

6) Vidal O, Valentini M, Espert JJ, et al. Laparoendoscopic singlesite cholecystectomy: a safe and reproducible alternative. J Laparoendosc Adv Surg Tech A 2009;19:599-602.

7) Curcillo PG, 2nd, Wu AS, Podolsky ER, et al. Single-port-access (SPA) cholecystectomy: a multi-institutional report of the first 297 cases. Surg Endosc 2010;24:1854-1860.

8) Rivas H, Varela E, Scott D. Single-incision laparoscopic cholecystectomy: initial evaluation of a large series of patients. Surg Endosc 2010;24:1403-1412.

9) Podolsky ER, Curcillo PG, 2nd. Reduced-port surgery: preservation of the critical view in single-port-access cholecystectomy. Surg Endosc 2010;24:3038-3043.

10) Edwards C, Bradshaw A, Ahearne P, et al. Single-incision laparoscopic cholecystectomy is feasible: initial experience with 80 cases. Surg Endosc 2010;24:2241-2247.

11) Morel P, Hagen ME, Bucher P, Buchs NC, Pugin F. Robotic single-port cholecystectomy using a new platform: initial clinical experience. J Gastrointest Surg 2011;15:2182-2186.

12) Konstantinidis KM, Hirides P, Hirides S, Chrysocheris P, Georgiou M. Cholecystectomy using a novel Single-Site((R)) robotic platform: early experience from 45 consecutive cases. Surg Endosc 2012;26:2687-2694.

13) Kroh M, El-Hayek K, Rosenblatt $\mathrm{S}$, et al. First human surgery with a novel single-port robotic system: cholecystectomy using the da Vinci Single-Site platform. Surg Endosc 2011;25:35663573.

14) McCaffery M, Pasero C. Pain : clinical manual. 2nd ed. St. Louis: Mosby; 1999. p.19.

15) Portenoy RK, Kanner RM. Definition and Assessment of Pain. In: Portenoy RK, Kanner RM, editors. Pain management : theory and practice. Philadelphia: F.A. Davis; 1996.

16) Chiruvella A, Sarmiento JM, Sweeney JF, Lin E, Davis SS, Jr. Iatrogenic combined bile duct and right hepatic artery injury during single incision laparoscopic cholecystectomy. Jsls 2010;14:268271.

17) Chang SK, Tay CW, Bicol RA, Lee YY, Madhavan K. A casecontrol study of single-incision versus standard laparoscopic cholecystectomy. World J Surg 2011;35:289-293.

18) Hirano $Y$, Watanabe $T$, Uchida $T$, et al. Single-incision laparoscopic cholecystectomy: single institution experience and literature review. World J Gastroenterol 2010;16:270-274.

19) Colon MJ, Telem D, Mermelstein J, Weber KJ, Divino CM, Chin E. Completely intracorporeal retraction of the gallbladder for laparoendoscopic single site (LESS) surgery. Surg Laparosc Endosc Percutan Tech 2011;21:e1-3.

20) Reibetanz J, Wierlemann A, Germer CT, Krajinovic K. A novel technique for fundal retraction of the gallbladder in single-port cholecystectomy. J Laparoendosc Adv Surg Tech A 2011;21:427429.

21) Morel P, Buchs NC, Iranmanesh P, et al. Robotic single-site cholecystectomy. J Hepatobiliary Pancreat Sci 2014;21:18-25. 